\title{
Photodynamic therapy for dysplastic Barrett's oesophagus: a prospective, double blind, randomised, placebo controlled trial
}

\author{
R Ackroyd, N J Brown, M F Davis, T J Stephenson, S L Marcus, C J Stoddard, \\ A G Johnson, M W R Reed
}

Division of Surgical and Anaesthetic Sciences, University of Sheffield, Sheffield, UK

R Ackroyd

N J Brown

C J Stoddard

A G Johnson

M W R Reed

Department of Medical Physics, Central Sheffield

University Hospitals, Glossop Road, Sheffield S10 2JF, UK

M F Davis

Department of Histopathology, Central Sheffield

University Hospitals, Glossop Road, Sheffield S10 2JF, UK $\mathrm{T} J$ Stephenson

DUSA

Pharmaceuticals Inc., 400 Columbus Avenue, Valhalla, NY 10595, USA

S L Marcus

Correspondence to: Professor M W R Reed, Division of Surgical and Anaesthetic Sciences, K Floor, Royal Hallamshire Hospital, Glossop Road, Sheffield S10 2JF, UK. m.w.reed@sheffield.ac.uk

Accepted for publication 9 May 2000

\begin{abstract}
Background and aims-Photodynamic therapy (PDT) is a treatment in which cell damage is achieved by the action of light on a photosensitising agent. We have assessed the potential use of PDT in the ablation of Barrett's oesophagus.

Methods-Thirty six patients with dysplastic Barrett's oesophagus receiving acid suppression medication with omeprazole were randomised to receive oral 5 -aminolaevulinic acid (ALA) $30 \mathrm{mg} / \mathrm{kg}$ or placebo, followed four hours later by laser endoscopy. Follow up endoscopy was performed at one, six, 12, and 24 months. Results-Of 18 patients in the ALA group, a response was seen in 16 (median decrease in area in the treated region $30 \%$; range $0-60 \%)$. In the placebo group, a decrease in area of $10 \%$ was observed in two patients with no change in 16 (median $0 \%$; range $0-10 \%$; treatment $v$ placebo, $\mathbf{p}<0.001)$. No dysplasia was seen in the columnar epithelium within the treatment area of any patient in the PDT group. However, in the placebo group, persistent low grade dysplasia was found in 12 patients $(p<0.001)$. There were no short or long term major side effects. The effects of treatment were maintained for up to 24 months.

Conclusions-This is the first randomised controlled trial of PDT for Barrett's oesophagus. It demonstrates that ALA induced PDT can provide safe and effective ablation of low grade dysplastic epithelium.

(Gut 2000;47:612-617)
\end{abstract}

Keywords: Barrett's oesophagus; photodynamic therapy; aminolaevulinic acid; protoporphyrin IX

The incidence of oesophageal adenocarcinoma is rapidly increasing in the western world. A major risk factor is Barrett's oesophagus, an acquired condition in which squamous mucosa is replaced by metaplastic columnar epithelium. The incidence has been estimated as 1 in 52 to 1 in 441 patient years. ${ }^{1-3}$ The risk is increased with dysplasia; in particular, high grade dysplasia (HGD) is considered a precursor of malignancy, and therefore an indication for oesophagectomy in suitable patients. ${ }^{34}$

Current therapeutic approaches aim to decrease oesophageal acid exposure by pharmacological or surgical means. However, despite the efficacy of medical treatment in symptom control and ulcer healing, there is little evidence that it produces regression of Barrett's epithelium. Results of antireflux surgery demonstrate regression in $40 \%$ of patients in one study, ${ }^{5}$ and squamous island formation in another, ${ }^{6}$ but others failed to show any regression..$^{7-9}$

Another approach is photodynamic therapy (PDT) which involves administration of a photosensitising drug followed by application of light to produce cell damage. The most commonly used photosensitiser is haematoporphyrin derivative $(\mathrm{HpD})$, which has been used in the treatment of early dysplasia and carcinoma in Barrett's oesophagus, although treatments have been complicated by stricture formation due to excess depth of tissue damage. ${ }^{10}$ Overholt reports the use of PDT using sodium porfimer and red light in the treatment of 100 patients with dysplasia and superficial carcinoma in Barrett's oesophagus. ${ }^{11}$ Mucosal ablation and squamous re-epithelialisation was seen in 75$80 \%$ of treated Barrett's mucosa, with complete elimination in 43 cases. Areas of dysplasia were eliminated in 78 patients and 10 of 13 malignancies were ablated. However, healing was associated with stricture formation in 34\%.

A novel approach to PDT is endogenous photosensitisation with aminolaevulinic acid (ALA), a naturally occurring compound in the haem biosynthetic pathway. This has no innate photosensitising properties but is metabolised to the photosensitive compound protoporphyrin IX (PpIX). This has several advantages over $\mathrm{HpD}$, in particular a reduced duration of photosensitisation and an affinity for epithelial tissues. This results in a more selective effect in the gastrointestinal mucosa, with less damage to underlying muscle thus reducing the risk of stricture and/or perforation.

ALA induced PDT has been used in the treatment of both dysplastic Barrett's oesophagus and oesophageal carcinoma. ${ }^{12-14}$ In a report of five patients with HGD in Barrett's oesophagus treated with ALA induced PDT, elimination of HGD and squamous regeneration was seen in all patients. There were no complications or recurrence of dysplasia after

Abbreviations used in this paper: PDT, photodynamic therapy; ALA, aminolaevulinic acid; LGD, low grade dysplasia; HGD, high grade dysplasia; $\mathrm{HpD}$, haematoporphyrin derivative; PpIX, protoporphyrin IX; Nd-YAG, neodymium-yttrium aluminium garnet; KTP, potassium titanyl phosphate; MPEC, multipolar electrocoagulation; ABPC, argon beam plasma coagulation. 
26-44 months of follow up but in two cases persistent non-dysplastic columnar glands were found beneath the neo-squamous epithelium. ${ }^{13}$

More recently, in a report of 32 patients with HGD $(n=10)$ or mucosal cancer $(n=22)$ in Barrett's oesophagus treated with ALA induced PDT, dysplasia was eradicated in all patients (10 of 10) and mucosal cancer in 17 of $22(77 \%)$ at a mean follow up of 9.9 (range 1-30 months). All tumours up to $2 \mathrm{~mm}$ in depth were completely ablated (17 of 17). There was no morbidity or mortality. ${ }^{14}$

While these studies demonstrate the feasibility of ALA induced PDT as treatment for Barrett's oesophagus, they are uncontrolled series and there have been no randomised controlled trials. This study aimed to assess the efficacy of PDT in the ablation of Barrett's oesophagus in a prospective, double blind, randomised, placebo controlled trial with observer blinded endoscopic and histological assessment of treatment response. The trial aimed to exclude placebo response due to laser light related hyperthermia or the effect of drug alone (omeprazole or ALA), and to eliminate avoidable sources of bias, such as observer error. We assessed not only the macroscopic effect on Barrett's mucosa but also the histological effects on dysplasia and the issue of "buried" glands seen in other studies, and attempted to establish the duration of treatment response. Treatment parameters were those established in a previous pharmacokinetic study of PpIX production after two different oral doses of ALA at three different time points. ${ }^{15} 16$

\section{Patients and methods}

After ethics committee approval was obtained, appropriate patients were sought from endoscopy and histopathology records. Patient recruitment took place throughout 1995, and eligibility was restricted to patients with low grade dysplasia (LGD) in circumferential Barrett's oesophagus of at least $3 \mathrm{~cm}$ in length who were receiving omeprazole. Patients were identified and the histology was re-examined by a single experienced gastrointestinal histopathologist (TJS) to confirm the diagnosis. Barrett's oesophagus was diagnosed only when specialised columnar epithelium (with intestinal metaplasia) was seen. ${ }^{17}$ Dysplasia was defined as neoplastic epithelium confined within the basement membrane in the absence of inflammation, and was classified as LGD according to accepted criteria. ${ }^{17} 18$ The histological diagnosis was confirmed on biopsy, no more than six weeks before treatment.

TREATMENT PROTOCOL

All treatments were performed as day cases. Patients were randomised to receive $30 \mathrm{mg} / \mathrm{kg}$ ALA or placebo. The randomisation was performed $1: 1$ by opening of one of a series of 36 previously sealed envelopes. This was done by pharmacy staff and the outcome was unknown to clinical staff and patients. ALA was dissolved in $50 \mathrm{ml}$ of orange juice or placebo was provided (orange juice alone). ALA is tasteless and so the treatment group could not detect the drug in their drink. Four hours after ALA or placebo administration, laser endoscopy was performed under intravenous sedation and analgesia (midazolam, pethidine, and propofol) administered by an anaesthetist. ALA dose and interval between ALA and light administration were selected on the basis of a previous dosimetric study. ${ }^{15} 16$

Once the patient was sedated, endoscopy was performed using an Olympus Q10 endoscope (Olympus Optical Co. Ltd, Tokyo, Japan). At this time, the length and percentage of the circumference of the oesophageal wall covered by Barrett's columnar epithelium was recorded, from which the area involved was calculated. All longitudinal measurements were taken from the upper incisor teeth (or gum) and confirmatory photographs were taken.

Laser light was generated by a copper vapour laser (Oxford 20 Watt, Oxford Lasers, Abingdon, UK) and delivered via a fibre with a diffuser tip (Laserscope Flex Cylinder Diffuser, PDT Systems Inc., Santa Barbara, California, USA). Uniform oesophageal light distribution was achieved using an applicator, consisting of a $14 \mathrm{~mm}$ diameter clear perspex cylinder attached to the end of an Atkinson tube introducer with a central channel $3 \mathrm{~cm}$ in length, housing the diffuser tip of the laser fibre. Before and after treatment, the light delivery system was calibrated using an integrating sphere, and throughout treatment light delivery was monitored by means of an isotropic detector fibre incorporated in the applicator.

The light applicator was lubricated and introduced over an endoscopically positioned guidewire into the oesophagus. It was positioned by means of $1 \mathrm{~cm}$ calibrated markings along the outer sheath so that the $3 \mathrm{~cm}$ "treatment window" was situated at the area of the oesophagus to be treated, as determined by measurement from the incisor teeth (or gums). Because of the size of the introducer it was not possible to check endoscopically the position of the light delivery system under direct vision.

All patients were treated with green light $(514 \mathrm{~nm})$ at a power density of $120 \mathrm{~mW} / \mathrm{cm}^{2}$ for a period of 500 seconds per $3 \mathrm{~cm}$ length. Temperature was monitored by a thermocouple in the applicator. Each therapy consisted of two separate treatments (distal then proximal; total treatment time 1000 seconds; energy density $60 \mathrm{~J} / \mathrm{cm}^{2}$ ) so that a total of $6 \mathrm{~cm}$ $(2 \times 3 \mathrm{~cm})$ length of oesophagus was treated, coinciding with the upper $6 \mathrm{~cm}$ of Barrett's mucosa. A $6 \mathrm{~cm}$ treatment represented complete treatment of Barrett's epithelium in 18 of $36(50 \%)$ patients.

If the Barrett's segment was between 3 and 6 $\mathrm{cm}$, two treatments were still performed. If tongues of columnar epithelium were observed above the area of circumferential Barrett's, the area to be treated was measured from the apex. This led to some areas of normal mucosa being included in the treatment area but this was necessary for complete treatment of the $6 \mathrm{~cm}$ length of Barrett's. If the length of Barrett's oesophagus was greater than $6 \mathrm{~cm}$, no further 
treatment was performed and any residual disease was left untreated. This was because the combination of the inflexibility and diameter of the introducing system and duration of treatment required to treat longer segments was considered inappropriate, particularly as the aim of the study was to study the effect of PDT rather than totally eradicate all traces of Barrett's oesophagus.

Following treatment, patients were given analgesia and antiemetics as required and were allowed to eat and drink as soon as they were able. They remained in hospital until dark and were then allowed home, with appropriate oral analgesia and a supply of antacids to take as necessary. Patients were advised to avoid bright light for 24 hours.

FOLLOW UP PROTOCOL

Throughout treatment and the whole of the follow up period (two years), patients were maintained on omeprazole $20 \mathrm{mg}$ daily. Follow up endoscopic assessments were performed at one, six, 12, and 24 months after treatment. The length and percentage of the involved circumference were recorded, from which the area was calculated.

At each visit, endoscopy was performed by two independent observers, neither of whom was aware of the patient's randomisation group, and both were blinded to the pretreatment assessment and to each other's assessment. Subsequent comparisons were made between the pre- and post-treatment assessments, and the two post-treatment assessments, from which the effect of treatment and degree of interobserver variation in endoscopic interpretation of Barrett's oesophagus was assessed. In each patient, the percentage change in area was recorded to the nearest $10 \%$, as it is not possible to quantify this to any greater degree. Only on final statistical analysis was the mean percentage area reduction calculated to the nearest $1 \%$.

Six biopsies were taken from the treated area for histological analysis at six, 12, and 24 months to confirm whether true regression from columnar to squamous epithelium had occurred and to assess the impact on dysplasia and the presence of buried glands. The biopsies were taken using standard endoscopic biopsy forceps and sent for histological analysis.
Sections were stained with haematoxylin and eosin and examined both by the routine hospital histopathology service and again by a single experienced gastrointestinal histopathologist (TJS) who was blinded to the other histological analyses and to the randomisation group of the patient.

\section{STATISTICAL ANALYSIS}

Before patients were recruited, a power calculation was performed using a commercially available software package (In Stat, Version 2.01, Graph Pub Software, San Diego, California, USA). It was calculated that to show a 30\% difference in outcome (as predicted from previous open studies) at a significance level (two sided) of $\mathrm{p}<0.05$ and a power of $90 \%, 16$ patients would be required in each group.

Patient demographics and pretreatment findings were analysed using either a $\chi^{2}$ or two tailed Mann-Whitney U test, as appropriate. The outcome of treatment was analysed in two ways. Both the proportion of responders and mean percentage of area regression were calculated for each of the two groups. Statistical analysis was performed using the SPSS for Windows (version 6.0) software package. To compare the proportion of patients responding in each group, a $2 \times 2$ comparison was made using a $\chi^{2}$ test. The percentage area regression was assessed by two tailed Mann-Whitney U test. Statistical significance was taken at $\mathrm{p}<0.05$.

\section{Results}

Of 70 patients initially thought to have LGD in Barrett's, the diagnosis was confirmed in 45 on expert pathology review. Of these, 36 patients (30 male, six female; median age 56 (range 30-71) years) agreed to enter the study, of whom 18 were given $30 \mathrm{mg} / \mathrm{kg}$ ALA and 18 received placebo. The two groups were demographically similar, with 15 men and three women in each and a median age of 56 (range 30-71) and 54 (range 44-68) years, respectively. Both had similar pretreatment lengths of Barrett's, with a median total length of $6 \mathrm{~cm}$ (range 4-15) and $7 \mathrm{~cm}$ (range 3-15), respectively (tables 1,2). Nine patients refused to take part in the study, five for family reasons and four claiming that they wished to see that

Table 1 Demographic details and treatment outcome in patients who received aminolaevulinic acid-photodynamic therapy

\begin{tabular}{|c|c|c|c|c|c|c|}
\hline Patient No & Sex & $\operatorname{Age}(y)$ & Pretreatment endoscopy findings & Post-treatment endoscopy findings & Post-treatment histology & Percentage area regression \\
\hline 1 & $M$ & 63 & $9 \mathrm{~cm}$ circumferential Barrett's & $7 \mathrm{~cm}$ circumferential Barrett's & Normal squamous & 30 \\
\hline 2 & $\mathrm{~F}$ & 30 & $6 \mathrm{~cm}$ circumferential Barrett's & 5 cm streaky Barrett's & Normal squamous & 50 \\
\hline 3 & $\mathrm{M}$ & 52 & $6 \mathrm{~cm}$ circumferential Barrett's & $4 \mathrm{~cm}$ Barrett's with islands++ & Normal squamous & 50 \\
\hline 4 & $\mathrm{M}$ & 58 & $13 \mathrm{~cm}$ circumferential Barrett's & $13 \mathrm{~cm}$ Barrett's with islands & Squamous/ Barrett's & 10 \\
\hline 5 & $\mathrm{M}$ & 49 & $6 \mathrm{~cm}$ circumferential Barrett's & $2 \mathrm{~cm}$ Barrett's $+3 \mathrm{~cm}$ streaks & Normal squamous & 60 \\
\hline 6 & M & 54 & $6 \mathrm{~cm}$ Barrett's $+1 \mathrm{~cm}$ tongue & $6 \mathrm{~cm}$ circumferential Barrett's & Squamous / Barrett's & 10 \\
\hline 7 & M & 53 & $14 \mathrm{~cm}$ Barrett's $+1 \mathrm{~cm}$ tongue & $13 \mathrm{~cm}$ Barrett's $+2 \mathrm{~cm}$ tongue & Normal squamous & 10 \\
\hline 8 & $\mathrm{M}$ & 58 & $4 \mathrm{~cm}$ Barrett's $+1 \mathrm{~cm}$ tongue & $3 \mathrm{~cm}$ Barrett's $+2 \mathrm{~cm}$ streaks & Normal squamous & 40 \\
\hline 9 & $\mathrm{~F}$ & 71 & $4 \mathrm{~cm}$ Barrett's + some islands & $3 \mathrm{~cm}$ Barrett's + islands++ & Normal squamous & 30 \\
\hline 10 & $\mathrm{M}$ & 53 & $4 \mathrm{~cm}$ Barrett's $+1 \mathrm{~cm}$ tongue & $3 \mathrm{~cm}$ Barrett's $+2 \mathrm{~cm}$ tongue & Normal squamous & 30 \\
\hline 11 & $\mathrm{M}$ & 64 & $6 \mathrm{~cm}$ circumferential Barrett's & $4 \mathrm{~cm}$ Barrett's $+2 \mathrm{~cm}$ tongue & Normal squamous & 30 \\
\hline 12 & M & 54 & $4 \mathrm{~cm}$ Barrett's $+1 \mathrm{~cm}$ tongue & $2 \mathrm{~cm}$ Barrett's $+2 \mathrm{~cm}$ tongue & Normal squamous & 50 \\
\hline 13 & $\mathrm{M}$ & 69 & $10 \mathrm{~cm}$ Barrett's $+2 \mathrm{~cm}$ tongue & $10 \mathrm{~cm}$ Barrett's $+2 \mathrm{~cm}$ tongue & Barrett's; no dysplasia & 0 \\
\hline 14 & M & 67 & $3 \mathrm{~cm}$ Barrett's $+2 \mathrm{~cm}$ tongue & $3 \mathrm{~cm}$ Barrett's $+2 \mathrm{~cm}$ tongue & Barrett's; no dysplasia & 0 \\
\hline 15 & $\mathrm{M}$ & 52 & $7 \mathrm{~cm}$ Barrett's $+2 \mathrm{~cm}$ tongue & $6 \mathrm{~cm}$ Barrett's $+2 \mathrm{~cm}$ tongue & Normal squamous & 10 \\
\hline 16 & $\mathrm{M}$ & 63 & $14 \mathrm{~cm}$ Barrett's + some islands & $11 \mathrm{~cm}$ Barrett's $+4 \mathrm{~cm}$ streaks & Normal squamous & 50 \\
\hline 17 & M & 54 & $7 \mathrm{~cm}$ Barrett's $+2 \mathrm{~cm}$ tongue & $5 \mathrm{~cm}$ Barrett's $+2 \mathrm{~cm}$ tongue & Normal squamous & 40 \\
\hline 18 & $\mathrm{~F}$ & 59 & $2 \mathrm{~cm}$ Barrett's $+3 \mathrm{~cm}$ streaks & $4 \mathrm{~cm}$ streaky Barrett's & Normal squamous & 60 \\
\hline
\end{tabular}


Table 2 Demographic details and treatment outcome in patients who received placebo

\begin{tabular}{|c|c|c|c|c|c|c|}
\hline Patient No & Sex & Age (y) & Pretreatment endoscopic findings & Post-treatment endoscopic findings & Post-treatment histology & Percentage area regression \\
\hline 1 & M & 65 & $6 \mathrm{~cm}$ circumferential Barrett's & $6 \mathrm{~cm}$ circumferential Barrett's & Barrett's with LGD & 0 \\
\hline 2 & M & 64 & $10 \mathrm{~cm}$ circumferential Barrett's & $10 \mathrm{~cm}$ circumferential Barrett's & Barrett's with LGD & 0 \\
\hline 3 & $M$ & 50 & $5 \mathrm{~cm}$ circumferential Barrett's & $5 \mathrm{~cm}$ circumferential Barrett's & Barrett's; no dysplasia & 0 \\
\hline 4 & M & 68 & $10 \mathrm{~cm}$ circumferential Barrett's & $10 \mathrm{~cm}$ circumferential Barrett's & Barrett's with LGD & 0 \\
\hline 5 & $\mathrm{M}$ & 60 & $10 \mathrm{~cm}$ Barrett's $+2 \mathrm{~cm}$ tongue & $9 \mathrm{~cm}$ Barrett's $+3 \mathrm{~cm}$ tongue & Barrett's with LGD & 10 \\
\hline 6 & M & 44 & $13 \mathrm{~cm}$ Barrett's $+1 \mathrm{~cm}$ tongue & $13 \mathrm{~cm}$ Barrett's $+1 \mathrm{~cm}$ tongue & Barrett's; no dysplasia & 0 \\
\hline 7 & M & 64 & $2 \mathrm{~cm}$ Barrett's $+2 \mathrm{~cm}$ tongue & $2 \mathrm{~cm}$ Barrett's $+2 \mathrm{~cm}$ tongue & Barrett's; no dysplasia & 0 \\
\hline 8 & M & 50 & $7 \mathrm{~cm}$ Barrett's $+2 \mathrm{~cm}$ streaks & $7 \mathrm{~cm}$ Barrett's $+2 \mathrm{~cm}$ streaks & Barrett's with LGD & 0 \\
\hline 9 & M & 48 & $8 \mathrm{~cm}$ Barrett's $+1 \mathrm{~cm}$ streaks & $8 \mathrm{~cm}$ Barrett's $+1 \mathrm{~cm}$ streaks & Barrett's with LGD & 0 \\
\hline 10 & $\mathrm{~F}$ & 63 & $3 \mathrm{~cm}$ Barrett's $+2 \mathrm{~cm}$ streaks & $2 \mathrm{~cm}$ Barrett's $+3 \mathrm{~cm}$ streaks & Barrett's with LGD & 10 \\
\hline 11 & M & 42 & $11 \mathrm{~cm}$ Barrett's $+1 \mathrm{~cm}$ streaks & $11 \mathrm{~cm}$ Barrett's $+1 \mathrm{~cm}$ streaks & Barrett's with LGD & 0 \\
\hline 12 & $M$ & 67 & $6 \mathrm{~cm}$ circumferential Barrett's & $6 \mathrm{~cm}$ circumferential Barrett's & Barrett's; no dysplasia & 0 \\
\hline 13 & $\mathrm{M}$ & 51 & $13 \mathrm{~cm}$ Barrett's $+2 \mathrm{~cm}$ streaks & $13 \mathrm{~cm}$ Barrett's $+2 \mathrm{~cm}$ streaks & Barrett's with LGD & 0 \\
\hline 14 & $M$ & 48 & $5 \mathrm{~cm}$ Barrett's $+1 \mathrm{~cm}$ streaks & $5 \mathrm{~cm}$ Barrett's $+1 \mathrm{~cm}$ streaks & Barrett's with LGD & 0 \\
\hline 15 & M & 57 & $2 \mathrm{~cm}$ Barrett's $+1 \mathrm{~cm}$ streaks & $2 \mathrm{~cm}$ Barrett's $+1 \mathrm{~cm}$ streaks & Barrett's with LGD & 0 \\
\hline 16 & M & 48 & $6 \mathrm{~cm}$ Barrett's $+1 \mathrm{~cm}$ streaks & $6 \mathrm{~cm}$ Barrett's $+1 \mathrm{~cm}$ streaks & Barrett's with LGD & 0 \\
\hline 17 & $\mathrm{~F}$ & 48 & $3 \mathrm{~cm}$ Barrett's $+2 \mathrm{~cm}$ tongue & $3 \mathrm{~cm}$ Barrett's $+2 \mathrm{~cm}$ tongue & Barrett's; no dysplasia & 0 \\
\hline 18 & $\mathrm{~F}$ & 68 & $7 \mathrm{~cm}$ circumferential Barrett's & $7 \mathrm{~cm}$ circumferential Barrett's & Barrett's; no dysplasia & 0 \\
\hline
\end{tabular}

the treatment was effective before agreeing to undergo several endoscopic examinations.

Of the 18 patients given photosensitiser, 16 $(89 \%)$ showed macroscopic evidence of regression at follow up endoscopy. The median reduction in area within the treated area was $30 \%$. Of the patients given placebo, a macroscopic response was seen in two $(11 \%)$. In these, the area regression was $10 \%$, producing an overall median response of $0 \%$ regression (tables 1,2). There was a statistically significant difference between the two groups (treatment $v$ placebo) in the number of patients responding $\left(\chi^{2}=21.8 ;\right.$ df $1, \mathrm{p}<0.001$, difference in proportions $78 \%, 95 \%$ confidence interval (CI) $56-100 \%$ ) and in median area regression (median area difference $30 \%, 95 \%$ CI 20$40 \% ; \mathrm{p}<0.001)$.

Histological examination of the biopsies taken at follow up endoscopy confirmed the macroscopic findings in all cases. In all 16 cases of regression in the treatment group, biopsies from the area of re-epithelialisation displayed normal squamous mucosa, with no evidence of either squamous dysplasia or underlying columnar epithelium. Moreover, biopsies of the remaining columnar epithelium in all 18 cases displayed no evidence of dysplasia in the area that had been treated with PDT. In the placebo group, biopsies from the area of reepithelialisation in the two cases showing some evidence of regression confirmed the appearance of normal squamous epithelium. Biopsies from the apparently unchanged columnar epithelium displayed Barrett's columnar mucosa in all cases. In 12 of 18 cases, there was persistent LGD but in six patients no evidence of dysplasia was seen (tables 1,2 ). With regard to the effect on dysplasia alone, there was therefore a statistically significant difference between the two groups $\left(\chi^{2}=18.0 ;\right.$ df 1 , $\mathrm{p}<0.001)$. In all cases, the underlying submucosa was identified, confirming that the biopsies taken represented full thickness samples.

SIDE EFFECTS

All 18 patients in the ALA treatment group experienced pain in the chest during the treatment (associated with restlessness and tachycardia). This discomfort persisted for 3-5 days following treatment and was aggravated by swallowing or coughing. However, only three patients required analgesia. One patient developed a mild skin rash after exposure to sunlight on the day after treatment. This resolved within 48 hours without the need for treatment. No patient complained of dysphagia at any stage following treatment.

\section{FOLLOW UP ASSESSMENT}

The interobserver variation in assessment of the area of Barrett's epithelium at the one month follow up endoscopy by the two independent observers was within $10 \%$ in 35 of 36 cases and differed by $30 \%$ in one case. Endoscopic assessments at six, 12, and 24 months after treatment were compatible within $10 \%$ in all cases to those at the one month assessment, with responses maintained over the 24 month follow up period. There were no cases of further progression or regression of the columnar epithelium or dysplasia in either group.

\section{Discussion}

When the present study was designed and implemented (1995), there were no published reports of the effect of ALA-PDT on Barrett's oesophagus. Therefore, we designed a double blind, randomised, placebo controlled trial to address this question. We did not aim to ablate all of the area of Barrett's change in every case for the following reasons:

(i) treatment of segments greater than $6 \mathrm{~cm}$ was considered inappropriate because of prolonged treatment times ( $>16$ minutes) due to the unsuitability of the introducing system used in this study. At this time, there was no commercially available balloon catheter;

(ii) confirmation that ALA-PDT is effective and safe in the ablation of Barrett's oesophagus in this type of study could be used to justify further studies designed to completely eradicate the Barrett's change, using a combination of longer flexible balloon devices and retreatment of unaffected areas. There are now several reports in the literature demonstrating the efficacy of ALA-PDT in the treatment of dysplastic Barrett's oesophagus. ${ }^{13}{ }^{14}$ However, the current study remains the only 
prospective, randomised, controlled study to date using treatment parameters established in a previous pharmacokinetic study. ${ }^{15} 16$

We demonstrated a significant difference between treatment and control groups in all parameters examined. There was a highly significant difference in the proportion of patients responding to treatment, with $90 \%$ and $10 \%$ responses in the treatment and control groups, respectively. There was also a significant reduction in columnar epithelial area in the treatment group. The reduction of Barrett's by only $30 \%$ of the treatment area and the patchy response within the treatment area in some patients could be seen as a weakness of the study. However, as previously stated, we did not aim to completely ablate the Barrett's change in all cases.

In most cases, PDT resulted in streaks or patches of columnar epithelium rather than complete circumferential ablation. The reason for this is unclear, but it may be due to the use of a solid state applicator with a fixed external diameter. The internal luminal diameter of the oesophagus varies within and between patients and is convoluted unless expanded. The streaks and patches of columnar epithelium remaining may be due to mucosal folds not eradicated by the applicator. Another disadvantage is the diameter of the applicator used in this study, requiring sedation for the introduction of the device. Oesophageal intubation would be easier with a more flexible device, which could be passed via the biopsy channel of an endoscope. The latter may also allow more accurate placement within the oesophagus, with less reliance on measurement of the distance from the incisor teeth, also permitting direct visualisation during treatment. The use of a balloon catheter may solve these problems, but such devices were not readily available at the onset of the study in 1995. Thus complete ablation of Barrett's oesophagus may be achieved far more readily using a combination of balloon light delivery devices, multiple treatments at each session, and repeated treatment sessions. The effect of increased doses of proton pump inhibitors should also be studied as this may account for some of the response seen in regression of Barrett's change and dysplasia in the placebo group.

Dysplasia was successfully eliminated in the treated area in the PDT group, including the two cases showing no obvious macroscopic response, while in the placebo group LGD persisted in two thirds of cases. The reason for the apparent elimination of dysplasia in one third of patients in the placebo group is unclear. It may have been due to continuing administration of omeprazole throughout treatment or to the natural history of LGD which can spontaneously regress in some cases. Indeed, it could be argued that it would be better to perform a trial such as this using only patients with HGD, but such patients are less common and would be harder to recruit. Equally, it may have been a sampling error at the time of the biopsy, with Barrett's epithelium containing only patchy dysplastic changes. At the time of planning this study, the value of systematic four quadrant biopsies in preference to multiple "random" biopsies was not recognised. However, despite the theoretical limitation of the biopsy regimen in the current study, dysplasia was detected in all patients before treatment and none after treatment in the PDT group. There was a significant difference between the two groups, indicating that ALA-PDT may cause regression of dysplasia without necessarily eradicating the associated Barrett's change. This unexpected finding has not been reported previously and requires further confirmation in future studies.

This trial confirmed the efficacy of ALA induced PDT in the treatment of dysplastic Barrett's oesophagus, the results in the treatment group being similar to those of other series. ${ }^{13}{ }^{14}$ A major drawback of treatment seen in previous studies has been the persistence of buried columnar glands beneath the neosquamous epithelium, with consequent implications for the remaining cancer risk. ${ }^{13}$ This was not encountered in our study. The reason for this may be due to the use of green light which, although it penetrates mucosa less well than red light, is entirely absorbed within the depth corresponding to the mucosa, possibly resulting in a greater PDT effect. ${ }^{19}$ Alternatively, it could be sampling error, but this is unlikely given that each patient had six biopsies on four occasions after treatment.

PDT is not the only form of ablative therapy currently under investigation for the treatment of Barrett's oesophagus. Thermal photocoagulation using neodymium-yttrium aluminium garnet $(\mathrm{Nd}-\mathrm{YAG})^{20}$ or potassium titanyl phosphate $(\mathrm{KTP})^{21} 22$ laser, multipolar electrocoagulation (MPEC), ${ }^{23}$ and argon beam plasma coagulation (ABPC) $)^{24}$ have been reported. In a report of 16 patients with non-dysplastic Barrett's oesophagus treated by KTP laser and acid suppression, mucosal ablation and squamous regeneration were seen in all cases. ${ }^{21}$ However, in 11 patients there was evidence of squamous regeneration over remaining Barrett's glands, and in nine patients squamous metaplasia was seen within Barrett's glands. In another study of 10 patients with Barrett's, complete mucosal ablation was achieved in all cases, but specialised mucosa was seen beneath the neo-squamous epithelium. ${ }^{22}$ In a report of 24 patients with Barrett's oesophagus (six with dysplasia) treated with ABPC, complete replacement of Barrett's mucosa by squamous epithelium was achieved in $13 .{ }^{24}$ However, these results were achieved with repeated treatments (median 2; range 1-7). Furthermore there were two oesophageal perforations (one fatal), a problem not seen in the present study.

Further studies are required to improve the efficacy of ALA induced PDT. The use of a balloon catheter may improve clinical outcome by eliminating folds in the oesophageal mucosa thereby improving intraoesophageal light distribution. This study has demonstrated that the treatment is safe and simple to perform. In light of this, a protocol of multiple treatments 
with the end point of producing complete ablation is required. Finally, randomised, placebo controlled clinical trials are needed to study the use of all types of ablative therapy and there will eventually be a need for prospective randomised trials to compare these different techniques.

This is the first prospective, double blind, randomised, placebo controlled trial of PDT to show effective ablation of dysplastic Barrett's oesophagus. It was a rigorous and unbiased assessment of this therapy and provides a clear indication of its potential. The majority of patients given photosensitiser and light showed evidence of macroscopic improvement following treatment, and in all cases there was apparent elimination of dysplasia. In contrast with other ablative therapies, or PDT with porphyrin based sensitisers, the low risk of complications renders this a viable treatment for dysplastic and possibly non-dysplastic Barrett's oesophagus.

This work was supported by a research grant from Trent Regional Health Authority. The authors wish to thank Regional Health Authority. The authors wish to thank
numerous clinical collaborators, including Mr A J Shorthouse, numerous clinical collaborators, including Mr A J Shorthouse, Dr J E Peacock for anaesthetic support, and to the staff of the Dr J E Peacock for anaesthetic support, and to the staff of the Day Case Unit, Endoscopy Unit and Pharmacy Manufacturing
Unit at the Royal Hallamshire Hospital, Sheffield for all their help and time spent assisting in this work.

1 Cameron AJ, Ott BJ, Payne WS. The incidence of adenocarcinoma in columnar-lined (Barrett's) esophagus. N Engl f Med 1985;313:857-9.

2 Spechler SJ, Robbins AH, Rubins HB, et al. Adenocarcinoma and Barrett's esophagus: an overrated risk? Gastroenterology 1984;87:927-33.

3 Miros M, Kerlin P, Walker N. Only patients with dysplasia progress to adenocarcinoma in Barrett's oesophagus. Gut 1991;32:1441-6.

4 Hamilton SR, Smith RRL. The relationship between columnar epithelial dysplasia and invasive adenocarcinoma arising in Barrett's esophagus. Am F Clin Pathol 1987;87: 301-12.

5 Brand DL, Ylvisaker JT, Gelfand M, et al. Regression of columnar esophageal (Barrett's) epithelium after antireflux surgery. $N$ Engl f Med 1980;302:844-8.

6 Skinner DB, Walther BC, Riddell RH, et al. Barrett's esophagus: comparison of benign and malignant cases. Ann Surg 1983;198:554-66.
7 Endo M, Kobayashi Kozu T. A case of Barrett's epithelialisation followed up for 5 years. Endoscopy 1974;6:48-51.

8 Dooner J, Cleator IG. Selective management of benign esophageal strictures. Am f Gastroenterol 1982;77:172-7.

9 Bremner CG. Barrett's oesophagus. In: Watson A, Celestin LR, eds. Disorders of the oesophagus: advances and controversies. London: Pitman, 1984:94-104.

10 Overholt B, Panjehpour M, Tefftellar E, et al. Photodynamic therapy for treatment of early adenocarcinoma in Barrett's esophagus. Gastrointest Endosc 1993;39:73-6.

11 Overholt BF, Panjehpour M, Haydek JM. Photodynamic therapy for Barrett's esophagus: follow-up in 100 patients. Gastrointest Endosc 1999;49:1-7.

12 Regula J, MacRobert AJ, Gorchein A, et al. Photosensitization and photodynamic therapy of oesophageal, duodenal, and colorectal tumours using 5-aminolaevulinic acid induced protoporphyrin IX - a pilot study. Gut 1995;36: induced $67-75$.

13 Barr H, Shepherd NA, Dix A, et al. Eradication of high-grade dysplasia in columnar-lined (Barrett's) oesophagus by photodynamic therapy with endogenously generated protoporphyrin IX. Lancet 1996;348:584-5.

14 Gossner L, Stolte M, Sroka R, et al. Photodynamic ablation of high-grade dysplasia and early cancer in Barrett's esophagus by means of 5-aminolevulinic acid. Gastroenterology 1998;114:448-55.

15 Ackroyd R, Roberts DJH, Vernon DI, et al. Photodynamic therapy (PDT) for Barrett's oesophagus: a dosimetric pilot study. Br F Surg 1996;83:1637.

16 Ackroyd R, Brown NJ, Reed MWR. Photodynamic therapy (PDT) for Barrett's oesophagus: establishing optimal treatment parameters. Eur f Surg Oncol 1996;22:551.

17 Haggitt RC. Barrett's esophagus, dysplasia, and adenocarcinoma. Hum Pathol 1994;25:982-93.

8 Riddell RH, Goldman H, Ransohoff DF. Dysplasia in inflammatory bowel disease: Standardized classification with provisional clinical implications. Hum Pathol 1983;14: 931-68.

19 Ackroyd R, Brown NJ, Stephenson TJ, et al. Ablation therapy for Barrett oesophagus: what depth of tissue destruction is needed? F Clin Pathol 1999;52:509-12.

20 Sampliner RE, Hixson LJ, Fennerty B, et al. Regression of Barrett's esophagus by laser ablation in an antacid environment. Dig Dis Sci 1993;38:365-8.

21 Barham CP, Jones RL, Biddlestone LR, et al. Photothermal laser ablation of Barrett's oesophagus: endoscopic and histological evidence of squamous re-epithelialisation. Gut 1997;41:281-4.

22 Gossner L, May A, Stolte M, et al. KTP laser destruction of dysplasia and early cancer in columnar-lined Barrett's esophagus. Gastrointest Endosc 1999;49:8-12.

23 Sampliner RE, Fennerty B, Garewal HS. Reversal of Barrett's esophagus with acid suppression and multipolar electrocoagulation: preliminary results. Gastrointest Endosc 1996;44:532-5.

24 Byrne JP, Armstrong GR, Attwood SEA. Endoscopic argon beam plasma coagulation in the restoration of squamous lining in Barrett's oesophagus. Endoscopy 1997;29:E33. 